\title{
QUALIDADE DE VIDA EM SUJEITOS RESPIRADORES ORAIS E ORONASAIS
}

\section{Life quality for mouth and oronasal breathing subjects}

\author{
Mirian Hideko Nagae ${ }^{(1)}$, Marcelo Corrêa Alves ${ }^{(2)}$, Renato Lyuti Kinoshita ${ }^{(3)}$, \\ Zélia Z L de Camargo Bittencourt ${ }^{(4)}$, Heloisa Gagliardo ${ }^{(5)}$
}

\begin{abstract}
RESUMO
Objetivo: investigar a qualidade de vida de sujeitos com respiração oral ou oronasal. Método: compuseram a amostra 49 voluntários, distribuídos em dois grupos: grupo de respiradores orais com 24 sujeitos e grupo de respiradores oronasais com 25 sujeitos, com faixa etária ente 18 e 38 anos de idade, de ambos os sexos. O protocolo utilizado foi o WHOQOL-BREF (versão em português) e o registro e análise dos dados foi realizado pelo sistema Statistical Analysis System. Resultados: os resultados obtidos foram submetidos aos testes de qui-quadrado de Mantel-Haenszele análise de variância. A qualidade de vida dos respiradores mistos se revelou inferior nos domínios ambiental, físico e psicológico em relação aos respiradores orais. Conclusão: com a detecção das diferenças obtidas, o estudo chama a atenção para a necessidade de aprofundar investigações sobre o perfil dos respiradores oronasais e sua relevância. Para que condutas como desconsiderar o grupo de respiradores oronasais ou agrupá-los como respiradores orais não sejam adotadas de forma inadequada.
\end{abstract}

DESCRITORES: Respiração; Qualidade de Vida; Saúde

\section{INTRODUÇÃO}

Respirar é condição vital para o ser humano e diversos estudos têm comprovado a maior adequação da respiração nasal ${ }^{1,2}$. Diante de comprometimentos como a insuficiência respiratória nasal, mecanismos compensatórios podem ocorrer. A respiração parcial (oronasal) ou integral

(1) Fonoaudióloga; Professora Doutora do Departamento de Desenvolvimento Humano e Reabilitação da Faculdade de Ciências Médicas da Universidade de Campinas-FCM/ UNICAMP, Campinas, São Paulo, Brasil.

(2) Engenheiro Agrônomo; Analista de Sistemas da Escola Superior de Agronomia Luiz de Queiroz da Universidade de São Paulo-ESALQ/USP, Piracicaba, São Paulo, Brasil.

(3) Fonoaudiólogo da Clinica de Otorrinolaringologia Rezende \& Paula, Campinas, São Paulo, Brasil.

(4) Assistente Social; Professora Doutora do Departamento de Desenvolvimento Humano e Reabilitaçãoda Faculdade de Ciências Médicas da Universidade de Campinas-FCM/ UNICAMP, Campinas, São Paulo, Brasil.

(5) Terapeuta Ocupacional; Professora Doutora do Departamento de Desenvolvimento Humano e Reabilitaçãoda Faculdade de Ciências Médicas da Universidade de Campinas-FCM/UNICAMP, Campinas, São Paulo, Brasil.

Conflito de interesses: inexistente pela boca (respirador oral) ${ }^{2,3}$ pode ser observada e estão associadas a um padrão de respiração ineficiente de causa obstrutiva ou não obstrutiva, viciosa ${ }^{4,5}$.

A respiração oral já é considerada por diversos autores como síndrome, a "Síndrome do Respirador Oral", caracterizada por alterações mecânicas das vias aéreas superiores e/ou alterações faciais ${ }^{2,6,7}$.

Nesses casos, alterações no desenvolvimento do sistema estomatognático ${ }^{2}$; posturais ${ }^{4,8}$; no sono ${ }^{7}$; comportamentais ${ }^{4,9}$; estéticas e funcionais ${ }^{4,10,11}$ e de aprendizagem ${ }^{12}$ podem ocorrer.

Tais comprometimentos respiratórios em geral são de instalação precoce ${ }^{7}$ e perpetuante onde os indivíduos muitas vezes se adaptam à situação e nem sempre percebem o impacto gerado na qualidade de vida.

Estudos epidemiológicos têm indicado que entre 10 e $25 \%$ da população geral apresentam comprometimentos respiratórios ${ }^{13}$, o que justifica considerar este, um problema de saúde pública ${ }^{2}$ por gerar prejuízos na qualidade de vida dos indivíduos ${ }^{14,15}$.

A maior parte dos estudos 7,8 com respiradores orais descreve as alterações do quadro de forma isolada, como eversão de lábio, sonolência diurna, 
falta de atenção, baixa concentração dentre outros, porém, as implicações no cotidiano desses indivíduos e principalmente dos respiradores oronasais tem sido pouco destacada na literatura ${ }^{14,16}$.

Os estudos não deixam claro, todavia, se há diferença entre o perfil do comportamento dos respiradores orais e oronasais. Os respiradores oronasais na maior parte das vezes não são considerados ${ }^{3,14}$ ou são agrupados como respiradores orais, como descrito na Síndrome do Respirador Oral ${ }^{4}$.

Com um olhar para a qualidade de vida, tem sido destacado na literatura que para assegurar a manutenção do bem-estar, é necessário cuidado constante. O qual inclui estratégias mediadoras entre as pessoas e o ambiente, com o objetivo de aumentar a participação dos sujeitos e da coletividade na modificação dos determinantes do processo saúde-doença, que inclui, entre muitos aspectos, os ligados aos hábitos de vida, lazer, trabalho, emprego, renda, educação e cultura. ${ }^{17}$.

Assim, compreende-se a qualidade de vida como uma categoria analítica central que favorece a promoção de ações integradoras e interdisciplinares ${ }^{18}$ e que decorre de uma construção subjetiva, multidimensional, constituída de elementos positivos e negativos ${ }^{19}$.

Tendo como premissa a concepção de saúde que considera o bem-estar físico, psíquico, social e não apenas a ausência de doença ${ }^{20,21}$ este estudo foi conduzido com o objetivo de analisar e comparar a qualidade de vida entre sujeitos adultos caracterizados como respiradores orais ou oronasais.

\section{MÉTODO}

Os participantes foram selecionados no Ambulatório de Motricidade Orofacial da Clinica de Fonoaudiologia da FCM/UNICAMP. A amostra foi constituída por 49 sujeitos, de ambos os sexos sendo 24 respiradores orais e 25 respiradores oronasais, com faixa etária entre 18 e 38 anos e diagnosticados por um otorrinolaringologista.

Os critérios de inclusão utilizados foram sujeitos que respirassem parcialmente ou integralmente pela boca e os de exclusão aqueles com alterações neurológicas, síndromes genéticas diagnosticadas, deformidades craniofaciais, e/ou que apresentassem histórico crônico de comprometimentos emocionais como ansiedade, depressão e compulsão.

A escolha do instrumento da pesquisa definiu-se por ser um protocolo validado para a população brasileira e ancorou-se no fato de que as pesquisas sobre qualidade de vida que utilizam instrumentos de medida podem colocar o Brasil em consonância com pesquisas internacionais que contribuem para o avanço metodológico e teórico na área e facilitar a compreensão da qualidade de vida em âmbito nacional ${ }^{22}$.

O instrumento utilizado para avaliação da qualidade de vida foi o World Health Organization Qualityof Life - WHOQOL-BREF ${ }^{23}$,(WHOQ) versão em português, composto por 24 questões divididas nos domínios: físico, psicológico, relações sociais e meio ambiente, além de duas questões gerais ${ }^{19}$. Cada domínio foi analisado isoladamente sendo que maiores escores correspondem a melhor qualidade de vida. Após a coleta dos dados, estes foram tabulados e analisados estatística e descritivamente.

O presente estudo foi aprovado pelo Comitê de Ética em Pesquisa da Faculdade de Ciências Médicas da Universidade Estadual de Campinas (FCM/UNICAMP) sob no 262/2010.

\section{Análise estatística}

A análise estatística dos dados se iniciou pela construção de tabelas de contingência para comparar as frequências e porcentagens de pessoas dos grupos oral e oronasal em categorias de escore dos domínios do WHOQOL-BREF. A aplicação do teste de qui-quadrado de MantelHaenszel para teste da hipótese de existência de associação linear entre os grupos de respiradores e os estratos dos escores dos domínios de qualidade de vida.

A comparação de médias de escore de qualidade de vida dos grupos oronasal e oral foi feita por meio de uma análise de variância com modelo apropriado para comparação de um fator com dois níveis. Um estudo preliminar evidenciou que a variável de resposta (escores dos domínios da qualidade de vida) seria melhor analisadas se adotada técnica que as tratasse como tendo distribuição lognormal. Foi adotada a técnica de estimação REML(RestrictedMaximumLikelihood) e a função de ligação identidade.

Os cálculos da análise de variância foram executados com apoio do procedimento (média e desvio padrão) Glimmix e a tabela de contingência e o teste de qui-quadrado de Mantel Haenszel com apoio do procedimento FREQ, ambos do sistema StatisticalAnalysis System ${ }^{24}$ (SAS).

\section{RESULTADOS}

$\mathrm{Na}$ Tabela 1 são apresentados os resultados referentes à distribuição dos grupos de respiradores orais e respiradores oronasais de acordo com os indicadores de qualidade de vida. 
Tabela 1 - Distribuição de frequências e porcentagens dos estratos de indicadores de qualidade de vida dos grupos estudados

\begin{tabular}{|c|c|c|c|c|c|c|c|}
\hline \multirow{2}{*}{ Domínio } & \multirow{2}{*}{ Grupo } & \multicolumn{5}{|c|}{ Estratos de indicadores de qualidade de vida } & \multirow{2}{*}{ Valor-p } \\
\hline & & $0-20$ & $20-40$ & $40-60$ & $60-80$ & $80-100$ & \\
\hline \multirow[t]{2}{*}{ Ambiental } & Oronasal & & $4(100,00)$ & $13(59,09)$ & $7(38,89)$ & $1(20,00)$ & 0,0140 \\
\hline & Oral & & $0(0,00)$ & $9(40,91)$ & $11(61,11)$ & $4(80,00)$ & \\
\hline \multirow[t]{2}{*}{ Psicológico } & Oronasal & & $4(80,00)$ & $10(83,33)$ & $10(43,48)$ & $1(11,11)$ & 0,0005 \\
\hline & Oral & & $1(20,00)$ & $2(16,67)$ & $13(56,52)$ & $8(88,89)$ & \\
\hline \multirow[t]{2}{*}{ Físico } & Oronasal & $1(100)$ & $5(83,33)$ & $11(84,62)$ & $8(38,10)$ & $0(0,00)$ & 0,0001 \\
\hline & Oral & $0(0,00)$ & $1(16,67)$ & $2(15,38)$ & $13(61,90)$ & $8(100,00)$ & \\
\hline \multirow[t]{2}{*}{ Social } & Oronasal & $0(0,00)$ & $3(100,00)$ & $10(55,56)$ & $9(52,94)$ & $3(30,00)$ & 0,1718 \\
\hline & Oral & $1(100)$ & $0(0,00)$ & $8(44,44)$ & $8(47,06)$ & $7(70,00)$ & \\
\hline
\end{tabular}

Teste Mantel-Haenszel Chi-Square $(p<0,005)$

Legenda: respirador oronasal: respiração oral e nasal, oral: respirador oral

Para verificar a existência de associação entre os grupos e os diferentes domínios de qualidade de vida explorados pelo WHOQOL-BREF, utilizou-se o teste de qui-quadrado de Mantel e Haenszel. Os resultados estatísticos demonstraram existência de associação entre os grupos estudados nos domínios ambiental, psicológico e físico, com valores de $p<0,05$, arbitrado como nível de significância estatística para o estudo. De acordo com as frequências obtidas nos diferentes escores verificou-se associação linear no domínio ambiental.

O teste de qui-quadrado de Mantel eHaenszeInos dá fortes indícios $(p<0,01)$ da existência de associação entre o grupo de respiração e os escores dos domínios psicológico e físico quantificados por meio do WHOQOL-BREF, que nos dá evidências $(p<0,05)$ da existência de associação linear entre os grupos e os escores do domínio ambiental.

Somente não foram encontrados indícios de associação no nível de significância de $5 \%$ no domínio social (p:0,1718), muito superior ao nível de significância arbitrado para o estudo.

A natureza ordinal usada no tratamento dos escores do WHOQOL-BREF revelou no domínio ambiental que $100,00 \%$ das pessoas com os escores mais baixos (20-40) são respiradores oronasais, proporção que se mantém mais alta $(59,09 \%)$ nos escores entre 40 e 60 . Nos escores mais altos, as proporções se inverteram, e nos escores entre 60 e 80 , houve uma minoria $(38,89 \%)$ de respiradores oronasais, diminuição que se consolida nos escore entre 80 e 100, onde há apenas $20 \%$ dos respiradores mistos.

Efeito similar foi observado quando se analisou o domínio psicológico onde um decréscimo da proporção de pessoas do grupo de respiradores oronasais nos maiores escores de qualidade de vida foi detectado. Enquanto que nos escores que revelam qualidades de vida inferiores (20 a 40 e 40 a 60) são observados respectivamente $80,00 \%$ e $83,33 \%$ de respiradores oronasais, quando analisados os escores mais altos de qualidade de vida a proporção de respiradores oronasais se mostra inferior à de respiradores orais com $43,48 \%$ no caso dos escores entre 60 e 80 e com $11,11 \%$ no caso dos escores entre 80 e 100.

O mesmo comportamento foi evidenciado no estudo do domínio físico, já que nos níveis mais baixos de qualidade de vida, de 0 a 20, 20 a 40 e 40 a 60 há respectivamente 100,00\%; 83,33\% e $84,62 \%$ de respiradores mistos, ou seja, os respiradores oronais aparecem em proporções maiores. Já nos níveis mais altos de qualidade de vida (60 a 80 e de 80 a 100) a proporção de respiradores oronasais é relativamente menor já que inferiores a $50 \%$ da amostra (38,10\% e 0,00\%).

Frente aos resultados obtidos, realizou-se a análise de variância com o objetivo de investigar os domínios isoladamente.

A análise de variância nos revelou existência de diferenças $(p<0,01)$ entre os grupos estudados em três domínios da qualidade de vida mensurados por meio do WHOQOL-BREF. Nos domínios físico, ambiental e psicológico, a média do escore qualidade de vida do respirador oronasal foi significantemente inferior à dos respiradores orais.

No domínio social a diferença foi não significante no nível de significância de 5\% ( $p: 0,2854)$ mas a média de escore do respirador oronasal também foi inferior a do grupo de respiradores orais. 
Tabela 2 - Média dos escore de qualidade de vida dos grupos de respiradores oronasal e oral

\begin{tabular}{|c|c|c|c|c|c|c|}
\hline \multirow[t]{2}{*}{ Grupo (domínio) } & \multirow[t]{2}{*}{ Média } & \multirow[t]{2}{*}{ DP } & \multicolumn{2}{|c|}{$\begin{array}{l}\text { Limites de confiança } \\
\text { da média }(95 \%)\end{array}$} & \multirow[t]{2}{*}{$\mathbf{F}$} & \multirow[t]{2}{*}{ Valor-p } \\
\hline & & & Superior & Inferior & & \\
\hline \multicolumn{7}{|l|}{ Ambiental } \\
\hline Oronasal & 54,1250 & 14,0544 & 59,9264 & 48,3236 & 9,19 & 0,0040 \\
\hline Oral & 65,3646 & 12,1527 & 70,4962 & 60,2330 & & \\
\hline \multicolumn{7}{|l|}{ Psicológico } \\
\hline Oronasal & 58,3333 & 15,4504 & 64,7110 & 51,9557 & 8,36 & 0,0058 \\
\hline Oral & 72,5694 & 14,9434 & 78,8795 & 66,2594 & & \\
\hline \multicolumn{7}{|l|}{ Físico } \\
\hline Oronasal & 51,0000 & 14,8160 & 57,1158 & 44,8842 & 22,08 & 0,0001 \\
\hline Oral & 73,8095 & 14,5422 & 79,9502 & 67,6689 & & \\
\hline \multicolumn{7}{|l|}{ Social } \\
\hline Oronasal & 60,6667 & 19,0212 & 68,5182 & 52,8151 & 1,17 & 0,2854 \\
\hline Oral & 68,0556 & 19,6077 & 76,3351 & 59,7760 & & \\
\hline
\end{tabular}

Teste de Análise de variância com modelo apropriado para experimentos com 1 fator (one-way ANOVA) e distribuição lognormal da variável de resposta

Legenda: oronasal: respirador oral e nasal, oral: respirador oral, DP: desvio padrão, F: frequencia

\section{DISCUSSÃO}

$\mathrm{Na}$ análise e comparação da qualidade de vida entre os respiradores orais e oronasais foi detectada uma diferença significante entre os grupos, em três dos quatro domínios de qualidade de vida analisados pelo WHOQOL-BREF.

Ao se investigar a frequência da qualidade de vida nos grupos detectou-se a existência de associação linear, onde na medida em que há um aumento da frequência indicando melhora de qualidade de vida o grupo de respiradores orais aumenta em proporção concomitantemente à diminuição da participação do grupo com respiração oral (Tabela 1).

Evidenciou-se com isso uma melhor qualidade de vida nos respiradores orais e pior nos respiradores oronasais, corroborando a hipótese fundamental do estudo de que os grupos apresentam diferenças significantes na qualidade de vida, e contrariando os estudos que consideram a inexistência de diferenças entre os grupos ${ }^{4}$ ou a irrelevância do grupo de respiradores oronasais ${ }^{3,14}$.

A partir dos resultados obtidos, destacou-se a importância de diferenciar cada grupo na população de sujeitos com ineficiência respiratória, uma vez que condutas específicas necessitam serem adotadas em cada situação devido ao perfil diferenciado dos grupos.

$\mathrm{Na}$ análise da qualidade de vida os domínios ambiental, físico e psicológico dos respiradores oronasais significantemente piores em relação aos respiradores orais (Tabela 2 ).

É interessante observar que o fato dos sujeitos respirarem parcialmente pela boca, não significa que a qualidade de vida seja melhor em relação àqueles que respiram integralmente pela boca, contrariando inclusive a hipótese inicial do trabalho.

O fato dos respiradores oronasais experimentarem a sensação de bem estar ao respirarem pelo nariz, mesmo que assistematicamente pode ser significativo nesses casos. Assim, a importância da "experiência" para que expectativas possam ser criadas, é referida em estudo sobre a qualidade de vida $^{25}$. O respirador oral que está fadado à condição de somente respirar pela boca, fatalmente acaba se adaptando as circunstâncias e dificilmente tem uma consciência crítica de seu estado.

Com isso nos respiradores mistos há provavelmente uma maior percepção de seus comprometimentos de saúde como dificuldade para respirar, falta de ânimo e necessidade do uso de medicamentos, caracterizados no domínio físico.

Ainda, na análise de variância evidenciou-se diferença significante entre os grupos no domínio psicológico, a proporção de respiradores oronasais nos escores que indicam pior qualidade de vida foi superior nesse domínio. Acredita-se que diante do reconhecimento das condições físicas negativas e adversas, o domínio psicológico também pode ser afetado, uma vez que alterações no rendimento, concentração, aparência física, humor e ansiedade 
podem ser desencadeados, como verificado em estudos com crianças e adolescentes ${ }^{14,26}$.

Já o fato do domínio ambiental ter sido afetado, provavelmente está relacionado com os fatores etiológicos alérgenos desencadeantes da obstrução nasal dos grupos, uma vez que a rinite alérgica foi considerada a maior causa das obstruções nasais na avaliação clínica dos sujeitos ${ }^{14,27}$.

$\mathrm{O}$ ambiente tem contribuído de maneira significante, devido aos avanços tecnológicos das últimas décadas, gerando o aumento vertiginoso da quantidade e variedade de contaminantes químicos eliminados no meio ambiente, além de mudanças abruptas das condições térmicas e condições insalubres de moradias ${ }^{21}$. Tais fatores não estão somente relacionados com o desencadeamento dos processos alérgicos como também com a perpetuação dos mesmos.
A percepção mais crítica dos respiradores mistos pode inclusive favorecer um maior enfrentamento da situação ${ }^{16}$, e ser um dos fatores que justifique o porque dos sujeitos não respirarem integralmente pela boca.

A detecção de diferenças estatisticamente significantes entre os grupos de respiradores orais e oronasais, apontam para a necessidade de continuidade dos estudos para melhor compreensão do perfil do comportamento de cada grupo. Os conhecimentos poderão ampliar e fundamentar ações fonoaudiológicas e interdisciplinares no âmbito da promoção da saúde.

\section{CONCLUSÃO}

Este estudo revelou que respiradores oronasais apresentam pior qualidade de vida que respiradores orais nos aspectos ambiental, psicológico e físico.

\begin{abstract}
Purpose: to investigate the life quality of subjects with mouth or oronasal breathing. Method: the sampling was composed of 49 volunteers distributed into 2 groups: mouth breathing subjects' group with 24 subjects and oronasal breathing subjects' group (mouth and nose) with 25 subjects, within the 18-38 age group, both genders. The WHOQOL-BREF (Portuguese version) protocol was used and the data analysis and recording were carried out using the Statistical Analysis System. Results: the obtained results underwent Mantel-Haenszel chi-square test and variance analysis. Life quality in oronasal breathing subjects was demonstrated to be better in environmental, physical and psychological domain in relation to mouth breathing subjects. Conclusion: considering the obtained differences, the study draws attention to the need for better investigating the profile of oronasal breathing subjects and its relevance, so that the conducts, such as disregarding the group of oronasal breathing subjects or gathering them as mouth breathing subjects, are not improperly adopted.
\end{abstract}

KEYWORDS: Respiration; Quality of Life; Health

\section{REFERÊNCIAS}

1. Krakauer LH, Guilherme A. Relationship between mouth breathing and postural alterations of children: a descriptive analysis. Int J Orofacial Myology. 2000; 26:13-23.

2. Menezes VA, Tavares RLO, Garcia AFG. Síndrome da respiração oral: alterações clinicas e comportamentais. Arquivos em Odontologia. 2009; 45(3): 160-5.

3. Queluz DP, Gimenes CMM. A síndrome do respirador bucal. Rev CROMG. 2000; 6(1):4-9.

4. Barros JR, Becker HM, Pinto JA. Evaluation of atopy among mouth-breathing pediatric patients referred for treatment to a tertiary care center. J Pediatr. 2006;82(6):458-64.

5. Bianchini AP, Guedes ZCF, Hitos S. Respiração oral: causa X audição. Rev CEFAC. 2009; 11(1):38-43

6. Oliveira AC, Lira dos Anjos CA, Silva EHAA, Menezes PL. Aspectos indicativos de envelhecimento facial precoce em respiradores orais adultos. Pró-Fono Revista de Atualização Científica. 2007;19(3):305-12.

7. Di Francesco RC, Passerotii G, Paulucci B, Miniti A. Respiração oral na criança: repercurssões diferentes de acordo com o diagnóstico, RevBrasOtorrinolaringol. 2004; 70(5):665-70. 
8. Ribeiro EC, Marchiori SC, Silva AMT. Electromyographic analysis of trapezius and sternocleidomastoideus muscles during nasal and oral inspiration in nasal and mouth breathing children. J of Electrom Kinesiology. 2002;12(4): 305-16.

9. Menezes VA, Leal RB, Pessôa RS, Pontes RMES. Prevalence and factors related to mouth breathing in school children at Santo Amaro ProjectRecife. Braz J Otorhinol. 2006;72:394-9.

10.Cintra CFSC, Castro FFM, Cintra PPVC. As alterações oro-faciais apresentadas em pacientes respiradores bucais. RevBrasAlerlmunopatol. 2000; 23(2):78-83.

11. Rodrigues HOSN, Faria SR, Paula FSG, Motta AR. Ocorrência de respiração oral e alterações miofuncionais orofaciais em sujeitos em tratamento ortodôntico. Rev CEFAC. 2005; 7(3):356-62.

12. Vera CFD, Conde GES, Wajnsztein R, Nemr $\mathrm{K}$. Transtornos de aprendizagem e presença de respiração oral em indivíduos com diagnóstico de transtornos de déficit de atenção/hiperatividade (TDAH). Revista CEFAC. 2006; 8(4):441-55.

13. Bousquet J, Van Cauwenberge P, Khaltaev N, Aria Workshop Group, World Health Organization. Allergic rhinits and its impacto on asma (ARIA). Workshop report.J AllergyClinImmunol. 2001; 108(5):147-334

14. Campanha SMA, Freire LMS, Fontes MJF. O impacto da asma, da rinite alérgica e da respiração oral na qualidade de vida de crianças e adolescentes. Rev. CEFAC. 2008;10(4):513-9.

15. Blaiss MS. Important aspects in management of allergic rhinitis: compliance, cost, and quality of life. Allergy Asthma Proc. 2003; 24(4):231-8.

16. Silva LF, Guedes MVC, Moreira RP, Souza ACC. Doença crônica: o enfrentamento pela família. Acta Paul Enf. 2002; 15(1):40-7.

17.Aerts D, Alves GG, La Salvia MW, Abegg C. Promoção de saúde: a convergência entre as propostas da vigilância da saúde e da escola cidadã. Cadernos de Saúde Pública [online]. 2004; 20(4):1020-8.

18.Penteado RZ, Pereira MTB. Qualidade de vida e saúde vocal de professores. Rev Saúde Pública. 2007;41(2):236-43.

19. Fleck MPA, Fachel O, Louzada S, Xavier M, Chachamovich E, Vieira G, et al. Aplicação da versão em português do instrumento abreviado de avaliação de qualidade de vida WHOQOL-bref. Rev Saúde Pública. 2000;34:178-83.

20. Minayo, MCS, Hartz ZMA, Buss PM. Qualidade de vida e saúde: um debate necessário. Revista Ciência e Saúde Coletiva. 2000; 5(1):7-18.

21.Gouveia N. Saúde e meio ambiente nas cidades: os desafios da saúde ambiental. Saúde e Sociedade. 1999;8(1):49-61.

22. Campos MO, Rodrigues Neto JF. Qualidade de vida: um instrumento para promoção de saúde. Revista Baiana de Saúde Pública. 2008;32(2):232-40.

23. WHOQOL-BREF.Whoqol Group. The World Health Organization quality of life assessment (WHOQOL): position paper from the World Health Organization. SocSci Méd. 1995; 10:1403-9. WHOQOL Group. Disponível em: http://www.ufrgs. $\mathrm{br} / \mathrm{psiq} /$ whoqol84.html

24. SAS Institude Inc. The SAS system release 9.2. SAS Institute Inc., Cary NC, 2008.

25. Carr AJ, Gibson B, Robinson PG. Is quality of life determined by expectations or experience? British Medical Journal. 2001;322:1240-3.

26. Junqueira $P$, Parro FM, Toledo MR, Araújo RLT, Di Francesco R, Rizzo MC. Conduta fonoaudiológica para pacientes com diagnóstico de rinite alérgica: relato de caso. Rev CEFAC. 2005; 7(3):336-9.

27. Wasilewska J, Kaczmarski M, Protas PT, Kowalczuk-Kryston M, Mazan B, Topczewska M. Sleep disorders in childhood and adolescence with special reference to allergic diseases. Pol Merkur Lekarski. 2009;26(153):188-93.

http://dx.doi.org/10.1590/S1516-18462012005000048

RECEBIDO EM: 29/09/2011

ACEITO EM: 15/11/2011

Endereço para correspondência:

Mirian Hideko Nagae

Av. Paulista 1195/152 - Bela Vista

São Paulo - SP

CEP: 01311/900

E-mail: mnagae@ @cm.unicamp.br 